\title{
Impact of High PV Penetration on Transient Stability — a Case Study on the U.S. ERCOT System
}

\author{
Abigail Till ${ }^{1}$, Shutang You ${ }^{1}$, and Yilu Liu ${ }^{1,2}$ \\ ${ }^{1}$ Department of Electrical Engineering and Computer Science \\ The University of Tennessee, Knoxville \\ Knoxville, Tennessee 37996, USA \\ Phone number: (+1) 865-974-1000, e-mail: aetill42@utk.edu, syou3@utk.edu, liu@utk.edu \\ ${ }^{2}$ Oak Ridge National Laboratory \\ 1 Bethel Valley Rd, Oak Ridge, TN 37830, USA \\ Phone/Fax number: 865-576-7658, e-mail: liu@utk.edu
}

\begin{abstract}
This study was performed to assess the impact of high photovoltaic (PV) penetration on transient stability using a series of hypothetical models of the ERCOT system. This was done by calculating the critical clearing time (CCT) when faulting various busses. Varying levels of PV penetration were used to study the system transient stability. For each case $15 \%$ wind penetration was also included.

It was found that under the PV volt/Var control with plant-level GE SolarControl settings, PV penetration at lower level can slightly improve the transient stability in the ERCOT system model. However, at approximately $54 \%$ penetration of renewables (39\% PV and 15\% wind), transient stability starts to show declining trend. This value has slight variance depending on PV distribution.
\end{abstract}

Key words. Transient stability, renewables, photovoltaic penetration.

\section{Introduction}

The influence of increasing renewable generation on power system stability has been the focus of recent years. For example, recent studies found that network-fault-induced wind turbine active power dips potentially represent a fundamental change in frequency stability risk [1]. Similar to wind power, the penetration of PV is increasing in many power systems due to concerns on environments and climate change. PV power plants have many unique physical features compared with conventional power plants and wind power generators. The increasing penetration of $\mathrm{PV}$ is changing many aspects on power system planning and operation.

Transient stability focuses on the capability of the system to keep synchronism under various disturbances in power systems. Typical theoretical study and demonstration on transient stability is usually based on a single machine and infinite bus system. Factors that influence transient stability include the generator loading condition, generator output during the fault, fault-clearing time, the infinite bus voltage magnitude (point of connection voltage magnitude), and etc. The dominant method for transient stability analysis for multi-machine power systems is time domain simulation based on numerical integration on differential equations and solving algebraic equations [2].

Some studies have already started to investigate the impact of PV on transient stability. Ref. [3] conducted a study on the WECC system and found that PV can have both detrimental and beneficial impact on the transient stability. The beneficial impact is generally associated with the fact that increased PV penetration usually represents more distributed generation, thus losing one transmission component will be less likely to lead to severe consequences. The detrimental impact comes from high PV penetration usually resulting in larger voltage perturbation.

Ref. [4] studied the system transient stability by integrating PV generation to the New England test system. It found that the PV power plants could significantly change the voltage profile after disturbance and make the system more vulnerable to stability problems. Ref. [5] studied the impact of PV on the IEEE-39 bus system when PV plants operate at unity power factor (zero reactive power output). Its simulation results showed that the higher PV penetration will impair the transient stability of the study system. Also based on the IEEE-39 bus system, the study in Ref. [6] found that if PV plants can provide voltage control during disturbance, voltage recovery during faults can be improved, thus enhancing transient stability. It is also demonstrated that under-voltage disconnection of PV can be detrimental to system stability since it will result in more significant excursions after disturbances.

The study in [7] investigated the stability of the Ontario system with large-scale PV under various scenarios, namely distributed units, centralized farms with and without voltage control capabilities. The result showed that the centralized PV farms working in voltage control or unity power factor mode, which are modeled as PV or 
PQ generators, have no major impact on the system transient stability. On the other hand, the distribution PV units, which are modelled as negative PQ loads, can significantly improve the voltage and transient stability. The underlying main reason of this improvement is that the increase of distributed PV corresponds to the reduction of load, thus improving transient stability metrics such as the critical clearing time. Ref. [8] displaced conventional generators by PV in a nine-bus model and found that the transient stability may be negatively affected by PV due to reduced inertia and higher generator reactance. This study also showed that the impact will be more serious if PV plants are simultaneously disconnected due to low voltage caused by disturbances. Ref. [9] utilized the IEEE 39-bus New England system to study the impact of PV on transient stability. Conducting small signal stability analysis and transient simulations, this study found that the factors pertaining to the detrimental or beneficial impact of PV on transient stability were the unit commitment and dispatch, and the protection/control strategy of PV during voltage swell or dip. Ref. [10] studied the impact of PV on a ninebus system and found that PV generation deteriorates the stability of the system, while the P-V control mode of PV generation is better for transient stability compared with the P-Q control mode. Ref. [11] adopted the New England-New York test system and found that the integration of PV increases the angular separation of synchronous generators.

Although some studies have investigated some basic impact factors of PV generation to transient stability, the understanding of high PV generation on actual power systems is still unclear due to the complexity and nonlinearity of PV control's interaction with power system electro-mechanic dynamics. This paper studied the impact of up to $80 \%$ renewable penetration in terms of instantaneous power (with $65 \% \mathrm{PV}$ instantaneous power) on the U.S. Electric Reliability Council of Texas (ERCOT) system model. Section 2 describes the high PV models of the ERCOT system. Section 3 presents the study results of high PV's impact on transient stability. The conclusion is given in Section 4.

\section{Model Overview}

In a previous work supported by U.S. Department of Energy Solar Energy Technologies Office [12-18], the high PV cases for the ERCOT system were developed in PSS ${ }^{\circledR} \mathrm{E}$ for $5 \%, 25 \%, 45 \%$, and $65 \%$ PV with $15 \%$ wind. The high PV cases were developed by replacing conventional synchronous generators with PV power plants, with a sequence of replacement starting from coal power plants, then gas power plants, and further nuclear power plants.

The model preserves general power flow information of a summer-peak snapshot of the ERCOT system, whereas the dynamics of generators are modelled using generic machine models and parameters. The system consists of 6,102 buses and 690 generation units. The frequency response of the base model was validated using several frequency events of ERCOT recorded in GridEye [13]. The total load is $74 \mathrm{GW}$. The inertia of the base case system is $2.2 \times \mathbf{1 0}^{\mathbf{5}} \mathbf{M V A} \cdot \mathbf{s}$.
PV power plants are modelled by the GE Solar PV dynamic model in $\mathrm{PSS}^{\circledR} \mathrm{E}$ [19]. PV applies volt/Var control and the plant-level supervisory control called GE SolarControl function. GE SolarControl defines a power factor range that determines real power levels at which control provides reactive power priority over real power. In addition, PV power plants are assumed to have enough fault ride through capacities that keep them online during faults.

\section{Impact of PV Penetration on Transient Stability}

\section{A. Critical Clearing Time with Slight PV Increase}

To study the impact of increasing PV penetration, conventional synchronous generators on the ERCOT system model are gradually replaced by PV penetration. Three synchronous generators are replaced by PV power plants in steps, replacing one generator in each step. To monitor the effect of PV on transient stability, the W4 generation unit on bus 80411 (with a 25.5 MW capacity) was replaced with a PV generation unit of the same capacity. A dynamic simulation was run for 1 second, and then a fault was applied at bus 8958, three buses away from the PV bus. The rotor angle was observed at bus 80411 to determine the critical clearing time (CCT). This was done for the base case with no PV, and then repeated as each machine was transformed to PV. The PV generators were on bus 80411, generators W4 (24 MW), W2 (25.5 MW), and W3 (24 MW), respectively.

Fig. 1-3 show the rotor angle when there was only one PV generator and the fault was cleared after $0.32 \mathrm{~s}, 0.33 \mathrm{~s}$, and $0.34 \mathrm{~s}$, respectively. When the fault is applied, the rotor angle is disturbed. If the rotor angle settles, then the case is stable. If the rotor angle diverges, then the case is unstable. The flat lines in Fig. 1-3 represent a state variable in the PV generation dynamic model, which keeps a constant value during the fault and therefore is ignorable. It can be observed from Fig. 1-3 that the addition of the PV generator slightly improves transient stability, since the CCT for the base case is $0.32 \mathrm{~s}$, and the CCT for the case with the PV generator is $0.33 \mathrm{~s}$. This can be seen by the "no PV" line diverging in Fig. 2 and then the "PV" line diverging in Fig. 3.

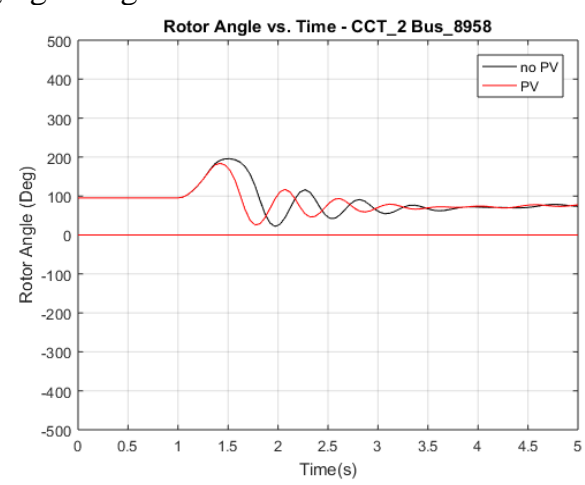

Fig. 1. Rotor angle when fault cleared after $0.32 \mathrm{~s}$. 


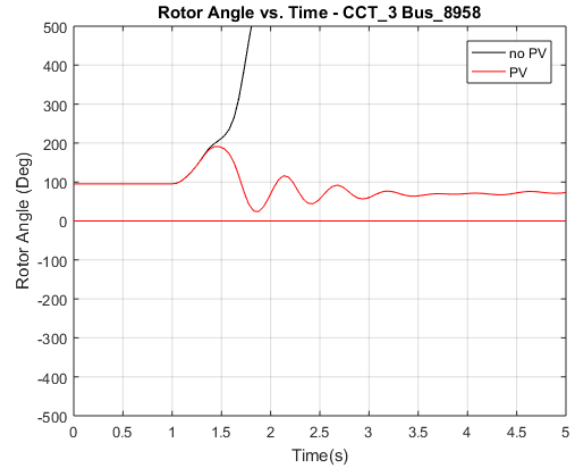

Fig. 2. Rotor angle when fault cleared after $0.33 \mathrm{~s}$.

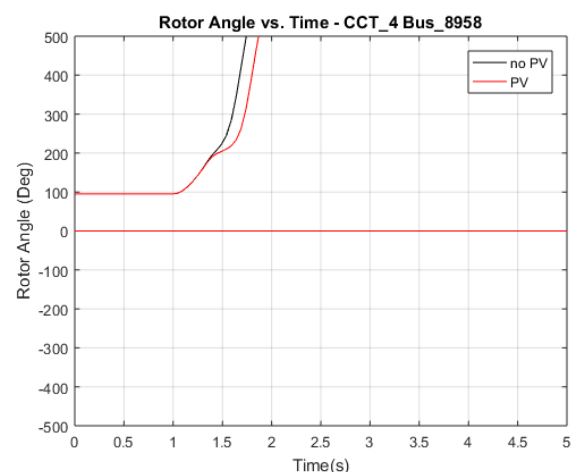

Fig. 3. Rotor angle when fault cleared after $0.34 \mathrm{~s}$.

A second PV generator was then added to bus 80411 and the simulation was run again. It was found that the CCT with two PV generators becomes $0.37 \mathrm{~s}$, which is a further improvement from the CCT with one PV generator added.

To test further, a third generator was replaced with a PV generator and the simulation was run another time. The CCT with three generators replaced with PV generators was found to be $0.44 \mathrm{~s}$.

Next the voltage was compared for the different cases at the CCT of the base case: $0.32 \mathrm{~s}$. This is shown in Fig. 4. It can be seen that as PV is added, the voltage level decreases more during the fault, but the voltage recovers more quickly.

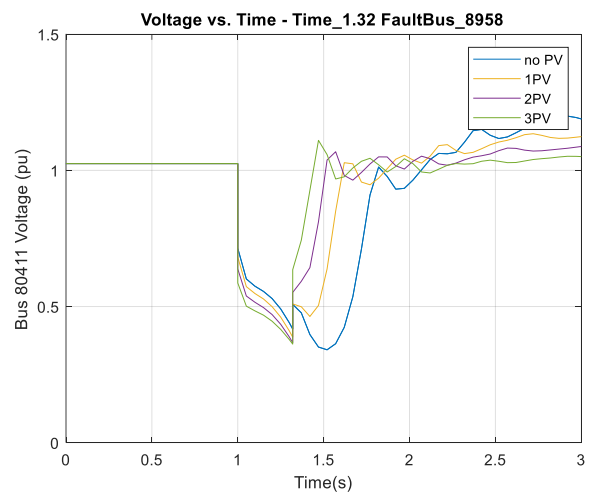

Fig. 4. Bus 80411 voltage for different amounts of PV generators.

Although the voltage level during the fault decreases as PV increases due to limited reactive power current, which results in faster rotor speed acceleration and smaller CCT, the fast voltage regulation of PV inverters can recover voltage more quickly compared with conventional synchronous generators after faults, leading to CCT increase. From the results, it can be seen the fast voltage recovery has a larger impact when PV generators increase in a local area.

B. Critical Clearing Time with Varying Percentages of PV

Once the stability was tested with a few PV generators, it was then tested for five different percentages of renewables in the ERCOT system $(5 \%, 25 \%, 45 \%$, and $65 \%$ PV with $15 \%$ wind each, and the base case without $\mathrm{PV}$ and wind power). A fault was applied to a bus on the system and the rotor angles were plotted to determine the CCT. The rotor angles on buses that contained only synchronous generators for the $80 \%$ renewable case were plotted for all of the cases, so they could all be compared equally. The fault was first applied at bus 240 , and then later the same test was run with the fault applied at bus 970 to confirm that the results would be similar with the fault on a different bus. To better view the results, the change in rotor angle was also plotted.

For the base case, with the fault on bus 240 the CCT was $0.25 \mathrm{~s}$, and with the fault on bus 970 the CCT was $0.38 \mathrm{~s}$. Fig. 5-6 show the rotor angle for when the fault on bus 240 was cleared at $0.25 \mathrm{~s}$ after the fault and at $0.26 \mathrm{~s}$, respectively. In Fig. 6 it is clear that the rotor angle diverges and therefore the system becomes unstable.

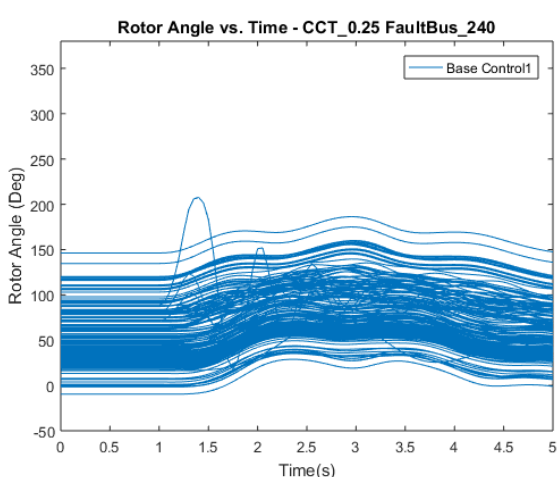

Fig. 5. Rotor angles when fault on bus 240 cleared at $0.25 \mathrm{~s}$.

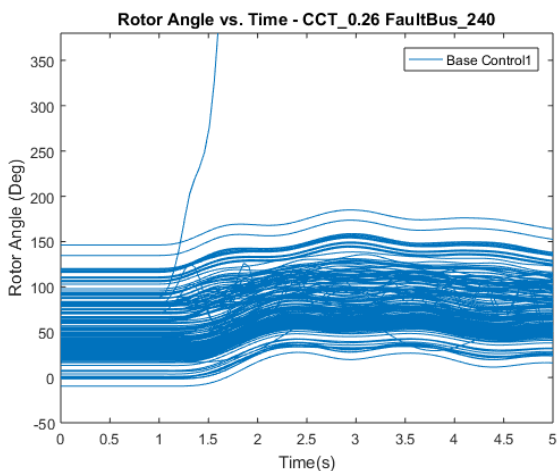

Fig. 6. Rotor angles when fault on bus 240 cleared at 0.26 s.

For the $20 \%$ renewable case (5\% PV $+15 \%$ wind), with the fault on bus 240 the CCT was $0.25 \mathrm{~s}$, and with the fault on bus 970 the CCT was $0.41 \mathrm{~s}$. With the fault on bus 240 , the CCT remained the same as the base case. With the fault 
on bus 970 , the CCT increased slightly with the increase in PV.

For the $40 \%$ renewable case $(25 \% \mathrm{PV}+15 \%$ wind $)$, with the fault on bus 240 the CCT was $0.25 \mathrm{~s}$, and with the fault on bus 970 the CCT was 0.39 s. With the fault on bus 240 , the CCT remained the same. With the fault on bus 970 , the CCT decreased slightly from the $20 \%$ renewable case with the increase in PV, although still an increased CCT from the base case.

For the $60 \%$ renewable case $(45 \% \mathrm{PV}+15 \%$ wind $)$, with the fault on bus 240 the CCT was $0.25 \mathrm{~s}$, and with the fault on bus 970 the CCT was $0.31 \mathrm{~s}$. With the fault on bus 240 , the CCT remained the same. With the fault on bus 970 , the CCT decreased by a tenth of a second from the $40 \%$ renewable case. This decrease also meant a CCT below that of the base case.

For the $80 \%$ renewable case $(65 \% \mathrm{PV}+15 \%$ wind $)$, with the fault on bus 240 the CCT was $0.01 \mathrm{~s}$, and with the fault on bus 970 the CCT was 0.115 s. For both cases, the CCT decreased significantly.

The CCT results were then graphed, and those results are shown in Fig. 7-8. From these results it was determined that more buses should be faulted to test if similar results would be achieved.

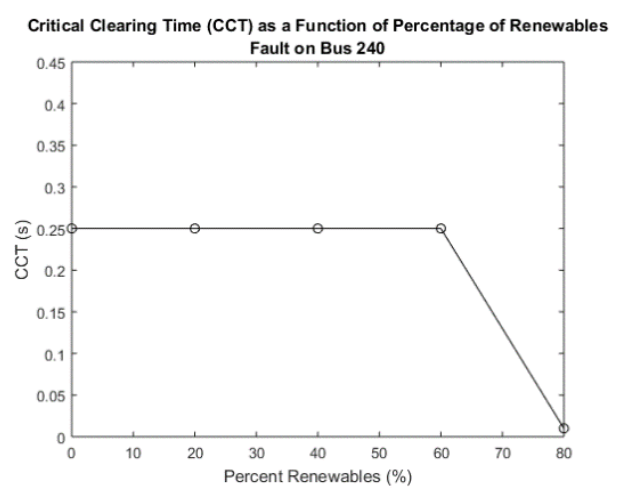

Fig. 7. CCTs for fault on bus 240 .

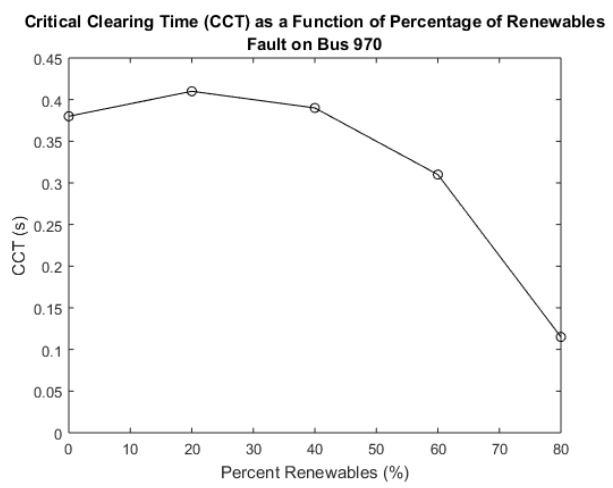

Fig. 8. CCTs for fault on bus 970 .

The CCT was then tested for faulting each bus $230 \mathrm{kV}$ and above. The results were similar to faulting buses 240 and 970, and a couple more examples are given in Fig. 9-10, showing buses 1685 and 3109 faulted, respectively. From these we can see that while the transient stability is sometimes slightly improved by added PV, by the time the system has reached higher levels such as $60 \%$ and $80 \%$ renewables the transient stability is much worse.

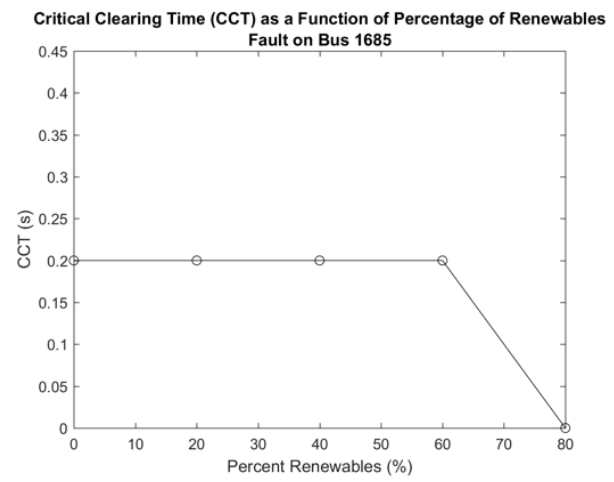

Fig. 9. CCTs for fault on bus 1685 .

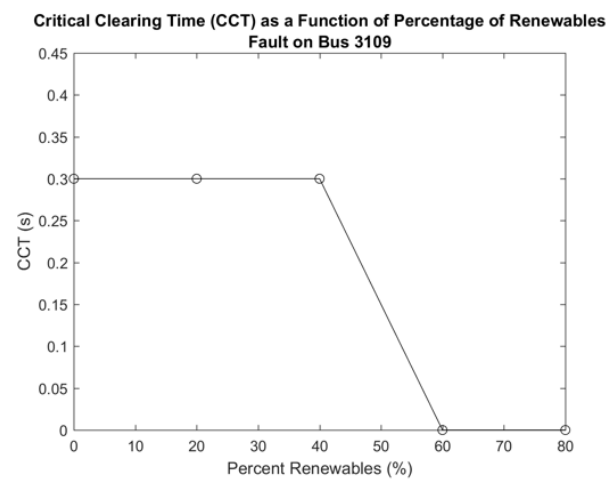

Fig. 10. CCTs for fault on bus 3109 .

C. Visualization of Critical Clearing Time for the ERCOT System

To better understand the impact of PV generation on overall transient stability of the ERCOT system, the CCTs of multiple high voltage buses were simulated. A color map of the ERCOT system was then created testing the CCT for faulting each bus $230 \mathrm{kV}$ and above. The maps were created for each renewable case: $0 \%, 20 \%, 40 \%$, $60 \%$, and $80 \%$. Fig. 11-15 contain the maps for each renewable case. The colors represent the CCT when the bus in that part of the ERCOT system is faulted. The CCT value for each faulted bus is mapped to its color using the color bar to the right of each map. For a CCT of 0 , the case did not converge.

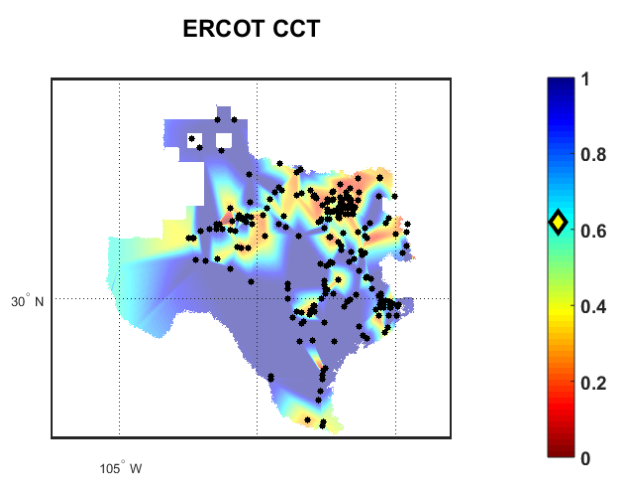

Fig. 11. Base case CCT map. 


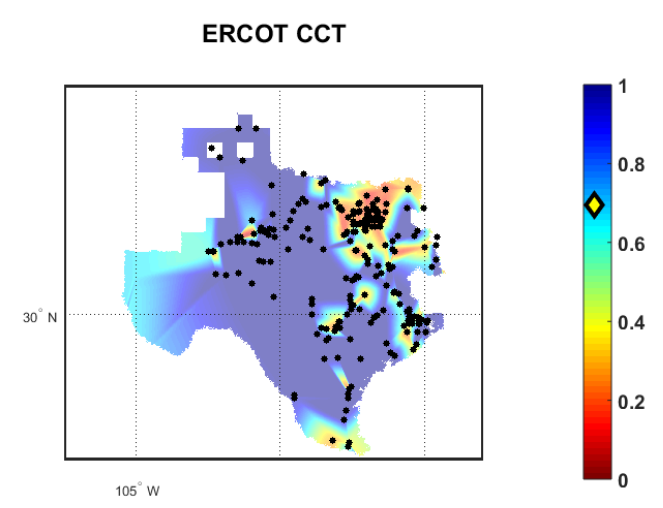

Fig. 12. 20\% renewables CCT map.

ERCOT CCT

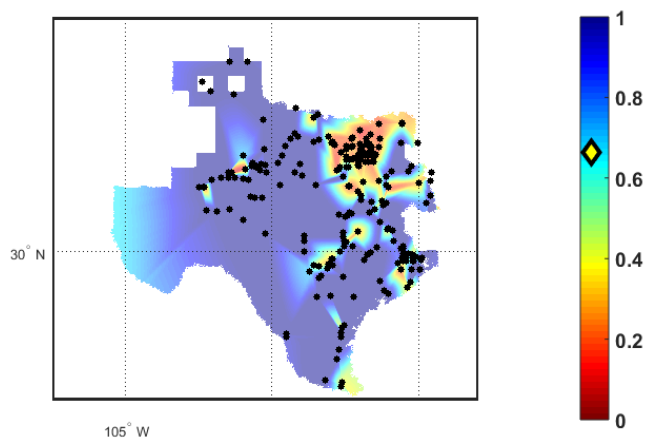

Fig. 13. $40 \%$ renewables CCT map.

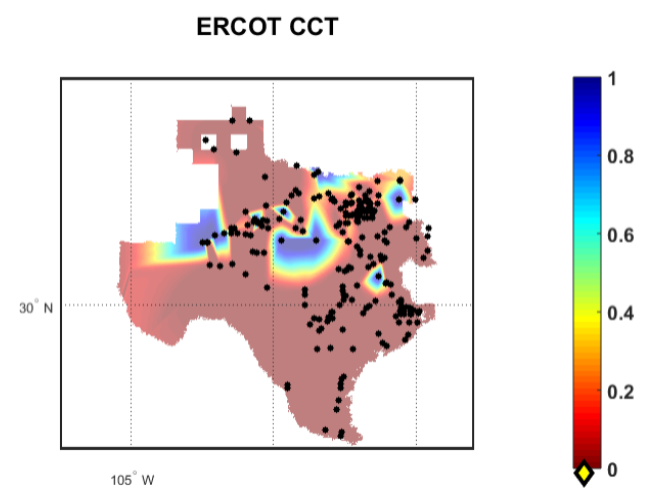

Fig. 14. 60\% renewables CCT map.

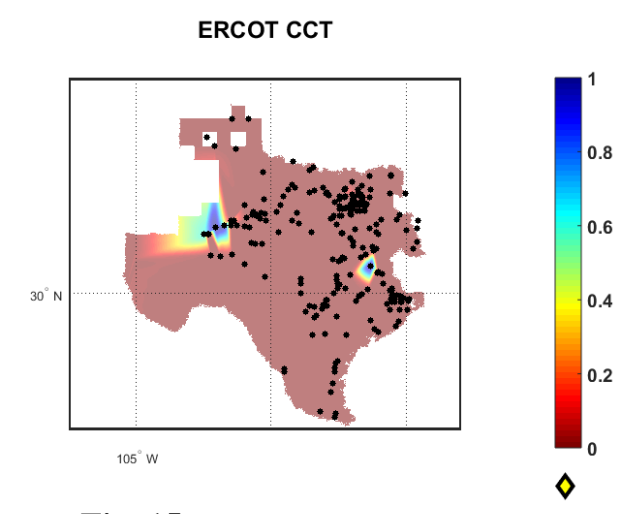

Fig. 15. 80\% renewables CCT map.

As can be seen from Fig. 11-15, the effect on the CCT of adding PV increases sharply between $40 \%$ and $60 \%$. The difference between the $40 \%-60 \%$ renewable cases were then investigated to determine what caused the CCT to decrease so significantly for most areas. The cases were broken down further to $45 \%, 50 \%$, and $55 \%$ renewables. The $50 \%$ and $55 \%$ renewables cases were similar to the $40 \%$ and $60 \%$ cases, respectively.

There was a difference of 13 generators between the $50 \%$ and $55 \%$ renewables cases. To further investigate at what point the CCT decreased, these generators were then changed to PV one at a time to determine the effect on the CCT. It was found that when the renewable percentage reached $54.2 \%$ that the CCT was affected significantly, as shown in Fig. 16-17. It was further found, however, that if different generators were replaced by PV, it was possible to reach a renewable percentage of $56.3 \%$ before the CCT was affected significantly.

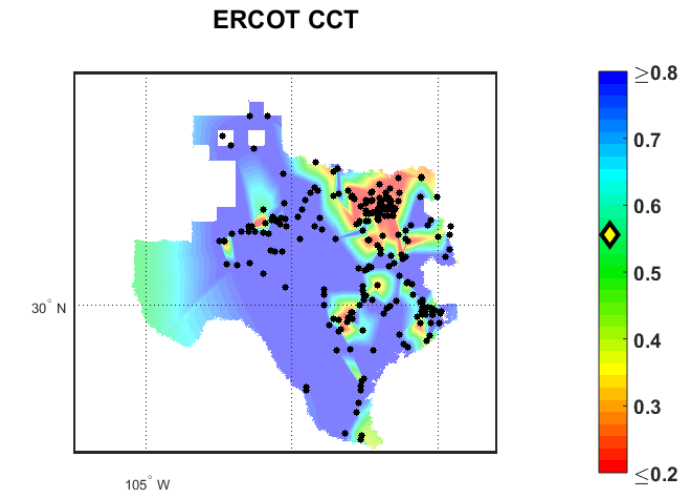

Fig. 16. 53.8\% renewable CCT map.

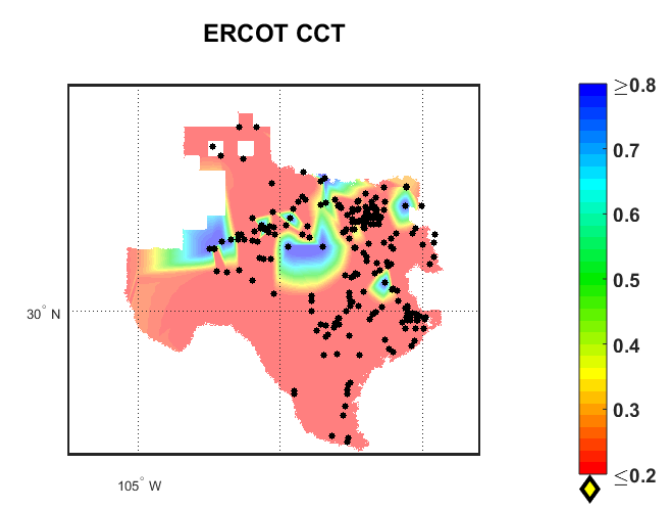

Fig. 17. $54.2 \%$ renewable CCT map.

This result shows that the impact of PV on ERCOT system frequency stability is non-linear. The incorporation of high PV penetration at the system level will fundamentally change the transient stability of a large-scale system and make the system very vulnerable to transient stability issues after a certain PV penetration point. In addition, simulation experience on model numerical convergence suggests that at high renewable penetration levels, static and dynamic reactive devices may be needed to deploy across the system, specifically, synchronous condensers in areas with low system strength. This phenomenon is also observed in other studies [20].

\section{Conclusion}

This study was performed to assess the impact of PV on transient stability of the ERCOT system. Study results show that under the volt/Var PV control and the plant level supervisory control (i.e. GE SolarControl) strategy, when 
adding just a few PV generators for the ERCOT system, adding the PV generators slightly improves stability of the system. For the system-level high renewables penetration scenarios developed in this hypothetical ERCOT system, when adding considerable amounts of renewables the stability may hold steady up to approximately $39 \%$ PV with $15 \%$ wind, after which the CCT drops considerably or the case does not converge which is indicated as zero CCT. These results reveal the highly nonlinearity of the impact of PV generation on transient stability.

In the future, the cause of divergence and instability will be investigated, to better assess how to determine the point of instability for other systems or under different operating conditions.

\section{Acknowledgements}

This work was supported in whole by the U.S. Department of Energy Solar Energy Technologies Office under Award Number 30844 and 34231. This work also made use of Engineering Research Center Shared Facilities supported by the Engineering Research Center Program (Award Number EEC-1041877) of the National Science Foundation and the U.S. Department of Energy under NSF, as well as the CURENT Industry Partnership Program. The authors are grateful to the U.S. Department of Energy technical managers who supported this work: Tassos Golnas, Hariharan Krishnaswami, and Guohui Yuan.

\section{References}

[1] O'Sullivan, Jon, Alan Rogers, Damian Flynn, Paul Smith, Alan Mullane, and Mark O'Malley. "Studying the maximum instantaneous non-synchronous generation in an island system - frequency stability challenges in Ireland." IEEE Transactions on Power Systems 29, no. 6 (2014): 2943-2951.

[2] K. Prabha, "Power system stability and control," Palo Alto Electrical Power Research Institute (EPRI)-McGraw-Hill, p. 202, 1994.

[3] S. Eftekharnejad, V. Vittal, G. T. Heydt, B. Keel, and J. Loehr, "Impact of increased penetration of photovoltaic generation on power systems," IEEE transactions on power systems, vol. 28, pp. 893-901, 2013.

[4] T. Alquthami, H. Ravindra, M. Faruque, M. Steurer, and T. Baldwin, "Study of photovoltaic integration impact on system stability using custom model of PV arrays integrated with PSS/E," in North American Power Symposium (NAPS), 2010, 2010, pp. 1-8.

[5] Y. T. Tan, "Impact on the power system with a large penetration of photovoltaic generation," 2004.

[6] S. Achilles, S. Schramm, and J. Bebic, Transmission system performance analysis for high-penetration photovoltaics: National Renewable Energy Laboratory, 2008.

[7] B. Tamimi, C. Cañizares, and K. Bhattacharya, "System stability impact of large-scale and distributed solar photovoltaic generation: the case of Ontario, Canada," IEEE transactions on sustainable energy, vol. 4, pp. 680-688, 2013.

[8] M. Yagami, N. Kimura, M. Tsuchimoto, and J. Tamura, "Power system transient stability analysis in the case of high-penetration photovoltaics," in PowerTech (POWERTECH), 2013 IEEE Grenoble, 2013, pp. 1-6.

[9] P. G. Bueno, J. C. Hernández, and F. J. Ruiz-Rodriguez, "Stability assessment for transmission systems with large utility-scale photovoltaic units," IET Renewable Power Generation, vol. 10, pp. 584-597, 2016.

[10] N. K. Roy, "Stability Assessment of Power Systems Integrated with Large-Scale Solar PV Units," Advances in Solar Photovoltaic Power Plants, pp. 215-230, 2016.

[11] R. Shah, N. Mithulananthan, and R. Bansal, "Oscillatory stability analysis with high penetrations of large-scale photovoltaic generation," Energy Conversion and Management, vol. 65, pp. 420429, 2013.

[12] S. You, Y. Liu, J. Tan, M.T. Gonzalez, X. Zhang, Y. Zhang, and Y. Liu, Comparative Assessment of Tactics to Improve Primary Frequency Response Without Curtailing Solar Output in High Photovoltaic Interconnection Grids. IEEE Transactions on Sustainable Energy, vol. 10, pp. 718-728, 2018.

[13] Y. Liu, S. You, J. Tan, Y. Zhang, and Y. Liu, Frequency response assessment and enhancement of the US power grids toward extrahigh photovoltaic generation penetrations-An industry perspective. IEEE Transactions on Power Systems, vol. 33, pp.3438-3449, 2018.

[14] S. You, G. Kou, Y. Liu, X. Zhang, Y. Cui, M.J. Till, W. Yao, and Y. Liu, Impact of high PV penetration on the inter-area oscillations in the US eastern interconnection. IEEE Access, vol. 5, pp.43614369, 2017.

[15] S. You, Y. Liu, G. Kou, X. Zhang, W. Yao, Y. Su, S.W. Hadley, and Y. Liu, Non-invasive identification of inertia distribution change in high renewable systems using distribution level PMU. IEEE Transactions on Power Systems, vol. 33, pp.1110 1112, 2017.

[16] S. You, Y. Liu, X. Zhang, M.T. Gonzalez, and Y. Liu. US Eastern Interconnection (EI) Electromechanical Wave Propagation and the Impact of High PV Penetration on Its Speed. In 2018 IEEE/PES Transmission and Distribution Conference and Exposition (T\&D), pp. 1-5, IEEE, April 2018.

[17] S. You, Y. Liu, Y. Liu, A. Till, H. Li, Y. Su, J. Zhao, J, Tan, Y, Zhang, M. Gong. Energy Storage for Frequency Control in High Photovoltaic Power Grids. In EUROCON 2019, pp. 1-6. 10.1109/EUROCON.2019.8861993.

[18] S. You, Y. Liu, X. Zhang, Y. Su, L. Wu, Y. Liu, and S.W. Hadley, Impact of high PV penetration on US eastern interconnection frequency response. In 2017 IEEE Power \& Energy Society General Meeting, pp. 1-5, IEEE. July. 2017

[19] Clark, K., Miller, N.W. and Walling, R., 2010. Modeling of GE solar photovoltaic plants for grid studies. General Electric International. Inc, Schenectady, NY, 12345.

[20] E. Rehman, M. Miller, J. Schmall, S.H. Huang. Dynamic Stability Assessment of High Penetration of Renewable Generation in the ERCOT Grid. 2018. Available at: http://www.ercot.com/content/wcm/lists/144927/Dynamic Stabili ty Assessment of High Penertration of Renewable Generatio... pdf 